According to the Theory of Ironic Processes of Mental Control (Wegner 1994) intentional thought suppression may paradoxically result in an unconscious activation of the suppressed thought (Deep Cognitive Activation). Chronic thought suppression may heighten the accessibility of suppressed thoughts to an extent that unwanted thoughts particularly under cognitive load - re-enter consciousness (Rebound-Effect) and influence (eating) behaviour.

Method: Obese children/teenagers ( $n$ 20; 11 boys and 9 girls) aged from 12 to 17 years $(M=14 \cdot 15$, sD $1 \cdot 53)$ participated in an experimental study during residential treatment. After being exposed to their favourite snacks, subjects were randomly assigned to one of two experimental conditions, a suppression condition ('do not think of your favourite snack') and an expression condition ('try to think of your favourite snack'). Simultaneously the amount of salivary secretion was measured. To test the accessibility of food-related concepts, subjects were instructed to complete several word fragment completion tasks under cognitive load (memorizing a 6-digit number). Finally, the amount of saliva secretion was measured for a second time.

Results: Suppression subjects showed both during suppression $(t(18)=2 \cdot 11, P=0 \cdot 05)$ and after suppression $(t(18)=3 \cdot 16, P<0 \cdot 01)$ significantly higher levels of saliva secretion compared with expression subjects.

Conclusions: Our findings clearly demonstrate that suppression of food-related thoughts ironically increases saliva secretion. Consequently restrictive food intake may be a counterproductive strategy and may be one explanation why obesity therapy is often quite ineffective.

\title{
56 - A one-year follow-up of a parent-led intervention for overweight children
}

\section{E Moens and C Braet}

Ghent University, Belgium
Introduction: Several reviews underscore the need to involve the parents in the treatment of paediatric obesity. However, there is still no clear insight into which specific components of family-based interventions are of particular significance and next to weight outcomes also behavioural outcomes merit more attention when assessing program effectiveness.

Method: A total of fifty families with overweight children (aged 6-12 years) were randomly allocated to a parent-led intervention group (cognitive behavioural training) or to a waiting list control group (Study 1). Afterwards, the parents of the waitlist control group followed the intervention as well. All children were followed in a follow-up study and were compared with a reference group of non-motivated families (Study 2).
Results: In Study 1, both the intervention group as well as the waitlist group showed a decrease in adjusted BMI over a 6-month period, although only significant for the intervention group. All children of Study 2 showed a decrease of $7 \%$ in adjusted BMI from pre- to one-year follow-up measurement, while the reference group showed an increase in adjusted BMI over that period. Parents reported positive changes in children's eating behaviour and in familial health principles.

Conclusions: The findings suggest that moderation of children's weight gain via parent groups is successful. Long-term follow-up is needed to reveal residual benefits of enhanced parenting skills on broader lifestyle changes.

Funding: The Johnson \& Johnson Fund for Health and Well-being of the King Baudouin Foundation.

\section{7 - Pediatric obesity: evidence from an integrated medical-psychological model}

\author{
P Cimbolli ${ }^{1}$, S Perfetto ${ }^{1}$, AM Caiazzo ${ }^{1}$, RE Papa ${ }^{1}$, A Mosca $^{1}$, A Piedimonte $^{1}$, \\ A Cafarotti ${ }^{1}$, A De Pascale ${ }^{2}$ and A Vania ${ }^{1}$
}

${ }^{1}$ Centro di Dietologia e Nutrizione Pediatrica, I Facolta di Medicina e Chirurgia, Italy: ${ }^{2}$ Dip.to di Scienze e Biotecnologie Medico-Chirurgiche, I Facolta di Medicina e Chirurgia - Polo Pontino, 'Sapienza' Universita di Roma, Italy 